\title{
TYPE-SPECIFIC LUMINOSITY FUNCTIONS OF NEARBY CLUSTERS OF GALAXIES
}

\author{
M. YAGI, K. SHIMASAKU, N. YASUDA, M. DOI AND S. OKAMURA \\ Department of Astronomy, University of Tokyo, Japan \\ N. KASHIKAWA \\ National Astronomical Observatory, Japan \\ AND \\ M. SEKIGUCHI \\ Institute for Cosmic Ray Research, University of Tokyo, Japan
}

We study the total luminosity function (LF) and the type-specific LF of 7 nearby clusters of galaxies (A1060, S805, A2063, A1736, A1644, A1631, and A754) using the R-band image $\left(1.0 \times 0.5 \mathrm{deg}^{2}\right)$ taken with our mosaic CCD camera mounted on 1-m telescope at the Las Campanas Observatory.

The data were reduced and analyzed with our own reduction software package. In each cluster, all objects larger than $2.5 \operatorname{arcsec}^{2}$ at 25 $\mathrm{mag} / \operatorname{arcsec}^{2}$ isophote are detected, and deblended if overlapped. We measure all detected objects, both isolated and deblended, and classify them into 3 classes, 'star', 'galaxy', and 'junk'. The magnitude limit of our galaxy sample is $R_{C} \sim 20 \mathrm{mag}$, which is imposed by the limit of the classification. About 2,000 galaxies are found in each cluster. According to the degree of luminosity concentration, we classify the galaxies into two types, $\mathrm{r}^{1 / 4}$-like and exponential-like. We find our classification for bright cluster galaxies is consistent with Dressler's (1980). Background number counts, both total and type-specific, are made from our blank field image. Then, we made total LF and type-specific LF for each cluster.

Results are the following. (1) The total-LFs are apparently different in shape from cluster to cluster. However, all but A1631 have a hump around $M_{R} \sim-19+5 \operatorname{logh}$. (2) The hump is clearly seen in the LFs of $\mathrm{r}^{1 / 4}$-like galaxies, while such hump is hardly seen in the LFs of exponential-like galaxies. (3) The LFs of exponential-like galaxies of the seven clusters are similar in shape, while that of $\mathrm{r}^{1 / 4}$-like galaxies have various shapes. The difference of the total LF found in (1) is mostly due to $\mathrm{r}^{1 / 4}$-like galaxies. 